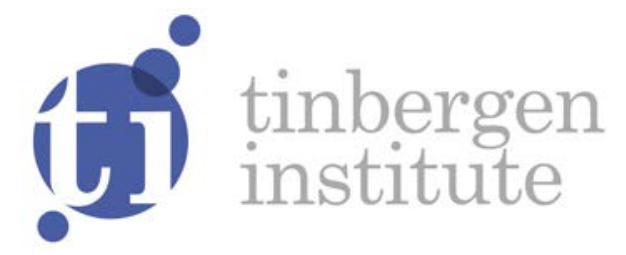

\title{
Measuring Financial Cycles in a Model-Based Analysis: Empirical Evidence for the United States and the Euro Area
}

\author{
Gabriele Galati \\ Irma Hindrayanto' \\ Siem Jan Koopman² \\ Marente Vlekke3
}

' De Nederlandsche Bank DNB, the Netherlands;

2 Faculty of Economics and Business Administration, VU University Amsterdam, and Tinbergen Institute, the Netherlands;

${ }^{3}$ Centraal Planbureau CPB, The Hague, the Netherlands. 
Tinbergen Institute is the graduate school and research institute in economics of Erasmus University Rotterdam, the University of Amsterdam and VU University Amsterdam.

More TI discussion papers can be downloaded at http://www.tinbergen.nl

Tinbergen Institute has two locations:

Tinbergen Institute Amsterdam

Gustav Mahlerplein 117

1082 MS Amsterdam

The Netherlands

Tel.: +31(0)20525 1600

Tinbergen Institute Rotterdam

Burg. Oudlaan 50

3062 PA Rotterdam

The Netherlands

Tel.: +31(0)10 4088900

Fax: +31(0)10 4089031 


\title{
Measuring Financial Cycles in a Model-Based Analysis: Empirical Evidence for the United States and the Euro Area
}

\author{
Gabriele Galati*, Irma Hindrayanto†, Siem Jan Koopman $\ddagger$ Marente Vlekke ${ }^{\S}$ \\ ${ }^{* \dagger}$ De Nederlandsche Bank, The Netherlands \\ $\ddagger$ Vrije Universiteit Amsterdam, The Netherlands \\ ${ }^{\ddagger}$ CREATES, Aarhus University, Denmark \\ ‡Tinbergen Institute Amsterdam, The Netherlands

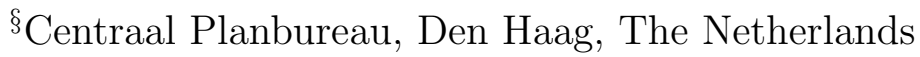

April 19, 2016

\begin{abstract}
We adopt an unobserved components time series model to extract financial cycles for the United States and the five largest euro area countries over the period 1970 to 2014. We find that credit, the credit-to-GDP ratio and house prices have medium-term cycles which share a few common statistical properties. We show that financial cycles are longer and more ample than business cycles, and that their length and amplitude vary over time and across countries.
\end{abstract}

Keywords: unobserved component time series model, Kalman filter, maximum likelihood estimation, band-pass filter, medium-term cycles.

JEL classification: C22, C32, E30, E50, E51, G01.

\footnotetext{
${ }^{*}$ Gabriele Galati, Economics and Research Division, De Nederlandsche Bank, The Netherlands.

${ }^{\dagger}$ Irma Hindrayanto, Economics and Research Division, De Nederlandsche Bank, The Netherlands.

${ }^{\ddagger}$ Siem Jan Koopman, Department of Econometrics \& OR, Vrije Universiteit Amsterdam, The Netherlands. CREATES, Aarhus University, Denmark. SJK acknowledges support from CREATES, Center for Research in Econometric Analysis of Time Series (DNRF78), funded by the Danish National Research Foundation, and from the European Union 7th Framework Programme (FP7-SSH/2007-2013, grant agreement 320270 - SYRTO) for financial support.

${ }^{\S}$ Marente Vlekke, Centraal Planbureau, Den Haag, The Netherlands
} 


\section{Introduction}

We explore a new approach to the measurement of financial cycles, and examine their main characteristics for the United States, Germany, France, Italy, Spain and the Netherlands. The financial cycle captures systematic patterns in the financial system that can have important macroeconomic consequences, see Borio et al. (2001). In recent years, several methods to measure the financial cycle have been proposed. These include variations of the Burns and Mitchell (1946) turning-point analysis, see e.g. Claessens et al. 2011, 2012), and nonparametric bandpass filters, see e.g. Aikman et al. (2015) and Schüler et al. (2015). Igan et al. (2009) and Hiebert et al. (2014) are among the papers that apply both approaches with the aim to reach more robust conclusions. The financial cycle is typically characterized by the co-movement of medium-term cycles in credit, the credit-to-GDP ratio and house prices; its peaks tend to coincide with onsets of financial crises (Drehmann et al. (2012)).

We consider a new approach to extract financial cycles based on a multivariate unobserved components time series model (UCTSM) for these three variables, see Harvey and Koopman (1997). The model is based on a joint decomposition of the three time series into long-term trends plus combinations of short- and medium-term cycles. We investigate whether the cycle processes for the individual series have the same frequencies and dynamic persistencies. While this approach has been applied extensively to business cycle analysis, see Valle e Azevedo et al. (2006) and references therein, there are only a few illustrations for financial variables, of which Koopman and Lucas (2005) and Chen et al. (2012) are examples. In comparison to non-parametric filters, we do not need prior assumptions on the length of the cycle, which is particularly convenient given our exploratory research on the financial cycle. Moreover, our model-based analysis relies on diagnostic statistics to investigate whether the model and the estimated trends and cycles are accurate and reliable.

\section{Data and modeling approach}

We aim to extract financial cycles from quarterly time series of credit, the credit-to-GDP ratio and house prices for the United States (US), Germany, France, Italy, Spain and the Netherlands, over the sample period from 1970 to 2014; the data sets are similar to the ones used in Drehmann et al. (2012). In our analysis, we have considered two definitions for credit: total credit and bank credit. We only present a selection of the results for total credit, all other results are available upon request from the authors. The time series are taken from the macroeconomic database of the Bank of International Settlements; they are deflated by the consumer price index and logarithms are taken except for the credit-to-GDP ratio.

We follow the steps in the analysis of Koopman and Lucas (2005): first we extract cycles from all time series based on an univariate UCTSM, and second, we verify whether the cycles in a multivariate UCTSM share common characteristics. Statistical diagnostic and test procedures are adopted to establish whether 'similar' cycles exist in the financial variables under consideration for the US and the five euro area (EA) countries. 


\subsection{Unobserved Components Time Series Models}

The observation vector of our three variables at time $t$ is denoted by $y_{t}$, for $t=1, \ldots, n$. The UCTSM in our study is formulated by a trend-cycle decomposition model for each variable equation $i$, that is

$$
y_{i t}=\mu_{i t}+\psi_{i t}+\varepsilon_{i t}, \quad \varepsilon_{i t} \stackrel{i . i . d .}{\sim} \mathcal{N}\left(0, \sigma_{\varepsilon, i}^{2}\right), \quad i=1,2,3
$$

where $y_{i t}$ is the $i$ th element of $y_{t}, \mu_{i t}$ represents the trend component, $\psi_{i t}$ represents the shortto medium-term cycle dynamics, and $\varepsilon_{i t}$ is the irregular that is normally distributed, with mean zero and variance $\sigma_{\varepsilon, i}^{2}$, and serially independent. The three components are unobserved. The trend associated with some variable is seemingly unrelated with the trends of the other two variables. This also applies to the cycle and the irregular. However, the covariances between the disturbances driving a particular component are typically non-zero and imply a dependence structure amongst the three variables and their dynamic characteristics.

A key part of the analysis is to determine the appropriate smoothness of the trend component, that is how much dynamic fluctuation in the variable $y_{i t}$ is assigned to the trend as opposed to the cycle. In terms of the $m$-order trend model of Harvey and Trimbur (2003), we determine the smoothness by the choice of $m$ in the trend specification $\mu_{i t}=\mu_{i t}^{\left(m_{i}\right)}$, with

$$
\begin{array}{cc}
\mu_{i, t+1}^{(k)}=\mu_{i t}^{(k)}+\mu_{i t}^{(k-1)}, & k=m_{i}, m_{i}-1, \ldots, 2, \\
\mu_{i, t+1}^{(1)}=\mu_{i t}^{(1)}+\zeta_{i t}, & \zeta_{i t} \stackrel{i . i . d .}{\sim} \mathcal{N}\left(0, \sigma_{\zeta, i}^{2}\right),
\end{array}
$$

where $\zeta_{i t}$ is the disturbance that drives the trends $\mu_{1 t}, \mu_{2 t}, \mu_{3 t}$, for $i=1,2,3$, since the covariance $\operatorname{cov}\left(\zeta_{i t}, \zeta_{j t}\right)$ can be non-zero, for $i \neq j=1,2,3$. In a frequency-domain analysis, a higher value for $m$ implies that the low-pass gain function will have a sharper cutoff. Hence the trend component becomes smoother as $m$ increases. when $m=2$, the trend reduces to an integrated random walk process. Notice that equation (2) implies $\Delta^{m} \mu_{i, t+1}^{(m)}=\zeta_{i t}$. For many macroeconomic time series, $m$ is typically set to 2 ; see, for example, Valle e Azevedo et al. (2006). This choice for $m$ is also adopted for the financial variables business failure rates and credit spreads in Koopman and Lucas (2005).

The cycle component $\psi_{i t}$ has the stochastic dynamic specification proposed by Harvey (1989) and is given by

$$
\left(\begin{array}{c}
\psi_{i, t+1} \\
\psi_{i, t+1}^{(*)}
\end{array}\right)=\phi_{i}\left[\begin{array}{cc}
\cos \lambda_{i} & \sin \lambda_{i} \\
-\sin \lambda_{i} & \cos \lambda_{i}
\end{array}\right]\left(\begin{array}{c}
\psi_{i t} \\
\psi_{i t}^{(*)}
\end{array}\right)+\left(\begin{array}{c}
\omega_{i t} \\
\omega_{i t}^{(*)}
\end{array}\right), \quad\left(\begin{array}{c}
\omega_{i t} \\
\omega_{i t}^{(*)}
\end{array}\right) \stackrel{i . i . d .}{\sim} \mathcal{N}\left(0, \sigma_{\omega, i}^{2} I_{2}\right),
$$

where the frequency $\lambda_{i}$ is measured in radians, $0 \leq \lambda_{i} \leq \pi$, and the persistence $\phi_{i}$ is the damping factor, $0<\phi_{i}<1$, for $i=1,2,3$. The period or length of the stochastic cycle $\psi_{i t}$ is given by $2 \pi / \lambda_{i}$. The cycle $\psi_{i t}$ is a stationary dynamic process. The disturbances $\left(\omega_{i t}, \omega_{i t}^{(*)}\right)^{\prime}$ drive the cyclical stochastic process and may be correlated with $\left(\omega_{j t}, \omega_{j t}^{(*)}\right)^{\prime}$, for $i \neq j=1,2,3$. All irregular, trend and cycle disturbances are serially and mutually uncorrelated, at all times and lags, but individually they can be correlated with their counterparts of the other two variables. The model is complete with appropriate initial conditions for trend $\mu_{i, 1}$ and cycle $\psi_{i, 1}$, for $i=1,2,3$, as discussed in Durbin and Koopman (2012). 


\subsection{Similar trends and cycles}

In our analysis, we investigate whether the trends and cycles can be treated as similar trends and cycles. In case of the trend, we verify whether the trend-order $m_{i}$ can be the same $m$, for $i=1,2,3$. In case of the cycle, we verify whether the frequency $\lambda_{i}$ and persistence $\phi_{i}$ can have respectively the same values for $i=1,2,3$. However, the scales of trends and cycles, as determined by the variances and covariances of the disturbances driving the components, can still be different under these 'similar' restrictions. The implications for the properties of similar cycles, both in the time- and frequency-domain analyses, are discussed in Harvey and Koopman (1997). Standard likelihood ratio tests can be used to verify whether the frequency and persistency parameters are equal amongst equations, after they are estimated from univariate UCTSMs.

\subsection{State space methodology}

Univariate and multivariate UCTSMs can be formulated in the general linear state space model as given by the observation equation $y_{t}=Z \alpha_{t}+\varepsilon_{t}$, with state vector $\alpha_{t}$, and the state updating equation $\alpha_{t+1}=T \alpha_{t}+\eta_{t}$, where $Z$ and $T$ are system matrices that determine the dynamic properties of $y_{t}$, and, together with the variance matrices for $\varepsilon_{t}$ and $\eta_{t}$, contain the static parameters of the model. The state vector contains the unobserved components $\mu_{t}$ and $\psi_{t}$, together with auxiliary variables such as $\mu_{t}^{(k)}$, for $k=1, \ldots, m-1$, and $\psi_{t}^{(*)}$ in equations (2) and (3). The various disturbances are placed in an appropriate manner in the vectors $\varepsilon_{t}$ and $\eta_{t}$. Further details of the trend-cycle model in state space form are provided by Harvey (1989).

Once the model is represented in state space form, the Kalman filter and related state space methods can be applied. We estimate the unknown static parameters by the method of maximum likelihood; numerical maximization requires the Kalman filter to compute the loglikelihood function. Given these estimates, we obtain prediction residuals for diagnostic checking and model evaluation from the Kalman filter. We obtain the smoothed estimates of the unobserved trend, cycle and irregular components from a smoothing method; see Durbin and Koopman (2012, Part I) for further details on the state space methodology.

\section{$3 \quad$ Empirical results}

For our empirical study, we have considered univariate and multivariate UCTSMs based on the decomposition (1) and for the three financial variables. We have carried out this analysis for US and five largest EA countries. The results of our empirical study can be summarized as follows. First, we find evidence of the existence of medium-term financial cycles in the US and EA. In particular, the majority of estimated financial cycles have lengths between 8 and 25 years, see Table 1. These lengths are clearly longer than the length of a business cycle which is typically between 6 and 8 years. Similar findings are also reported in Drehmann et al. (2012). Second, many financial cycles have larger amplitudes compared to those of typical business cycle. The amplitude can be measured by the range of the medium-term fluctuations which are presented in Figure 1. The amplitudes of our extracted financial cycles range between $10 \%$ and $20 \%$, with the exception of Germany. The business cycle amplitude 
is usually around 5\%; see also the discussion in Zarnowitz and Ozyildirim (2002). Hence we can conclude that financial cycles are distinct from business cycles.

Third, we do find evidence of significant heterogeneity between countries, in comparison with the US but also among the EA countries. For the EA, we observe clear differences between the financial cycles on the one hand of Germany and the Netherlands, and on the other hand those of France, Italy, and Spain; a similar distinction is revealed in Schüler et al. (2015). Whereas the former group has financial cycles of around 10 years, the latter group has longer financial cycles with a larger amplitude. These findings indicate that the financial sectors in different countries operate in different ways. Fourth, we do not only observe heterogeneity across countries, but also over time. The US financial cycles are much shorter and smaller in the pre-1985 period; see Drehmann et al. (2012) and Borio (2014) who also presented longer and more ample cycles for the US after 1985.

Table 1: Parameter estimates for total credit, credit-to-GDP ratio and house prices

\begin{tabular}{lrrrrrr}
\hline \multicolumn{1}{c}{ US } & Germany & Netherlands & France & Italy & Spain \\
\hline Univariate cycles & & & & & & \\
\hline$\phi-$ CR & 0.998 & 0.946 & 0.984 & 0.986 & 0.994 & 0.998 \\
$\phi-\mathrm{C} /$ GDP & 0.994 & 0.982 & 0.963 & 0.977 & 0.991 & 0.998 \\
$\phi-$ HPR & 0.996 & 0.973 & 0.987 & 0.996 & 0.993 & 0.993 \\
$p-$ CR & 14.56 & 7.78 & 10.27 & 12.13 & 17.53 & 13.08 \\
$p-$ C/GDP & 15.50 & 24.75 & 11.77 & 16.84 & 16.94 & 15.99 \\
$p-$ HPR & 14.41 & 10.11 & 10.41 & 15.08 & 12.35 & 14.51 \\
loglikelihood & 1754.2 & 1755.2 & 1479.9 & 1681.1 & 1412.1 & 1495.7 \\
AICc & -3482.5 & -3484.6 & -2934.0 & -3336.3 & -2798.1 & -2965.6 \\
BIC & -3446.1 & -3448.1 & -2897.5 & -3299.8 & -2763.0 & -2929.2 \\
\hline Similar cycles & & & & & & \\
\hline$\phi$ & 0.996 & 0.956 & 0.983 & 0.992 & 0.992 & 0.997 \\
$p$ & 14.61 & 9.78 & 10.53 & 14.66 & 14.67 & 14.31 \\
loglikelihood & 1753.5 & 1751.2 & 1478.5 & 1677.4 & 1407.9 & 1494.3 \\
AICc & -3490.2 & -3485.6 & -2940.2 & -3338.0 & -2798.9 & -2971.7 \\
BIC & -3465.5 & -3460.9 & -2915.5 & -3313.3 & -2774.2 & -2947.0 \\
\hline LR test & 1.4 & 8.0 & 2.8 & 7.3 & 8.4 & 3.0 \\
$\#$ observations & 180 & 180 & 180 & 180 & 164 & 180 \\
\hline
\end{tabular}

We report estimates of the persistency $(\phi)$ and the period ( $p=2 \pi / \lambda$, in years) parameters, for the univariate and multivariate UCTSM. The three equations and its parameters are indicated by $\mathrm{CR}$ - total credit, C/GDP - credit-to-GDP ratio, and HPR - house prices. The estimates are based on our country panel of quarterly data, for sample 1970-2014, except Italy 1974-2014. We further report the maximized loglikelihood value, the Akaike Information Criterion (AICc), with finite-sample correction, Schwarz's Bayesian Information Criterion (BIC), the likelihoodratio (LR) tests the hypothesis $H_{0}: \lambda_{H P}=\lambda_{C R}=\lambda_{C R / G D P}$ and $\phi_{H P}=\phi_{C R}=\phi_{C R / G D P}$, which is asymptotically $\chi^{2}$-distributed, with 4 degrees of freedom and critical value 7.78 for a $10 \%$ significance level.

Fifth, in accordance with the studies of Claessens et al. (2011, 2012), the extracted cycles from univariate UCTSMs share a number of important characteristics. In particular, for most countries, the estimates for persistence (measured by $\phi$ ) and length (measured by the period $2 \pi / \lambda)$ do not vary much across the three variables. It implies that time- and frequency- 
Figure 1: Extracted similar cycles for total credit, credit-to-GDP ratio, and house prices
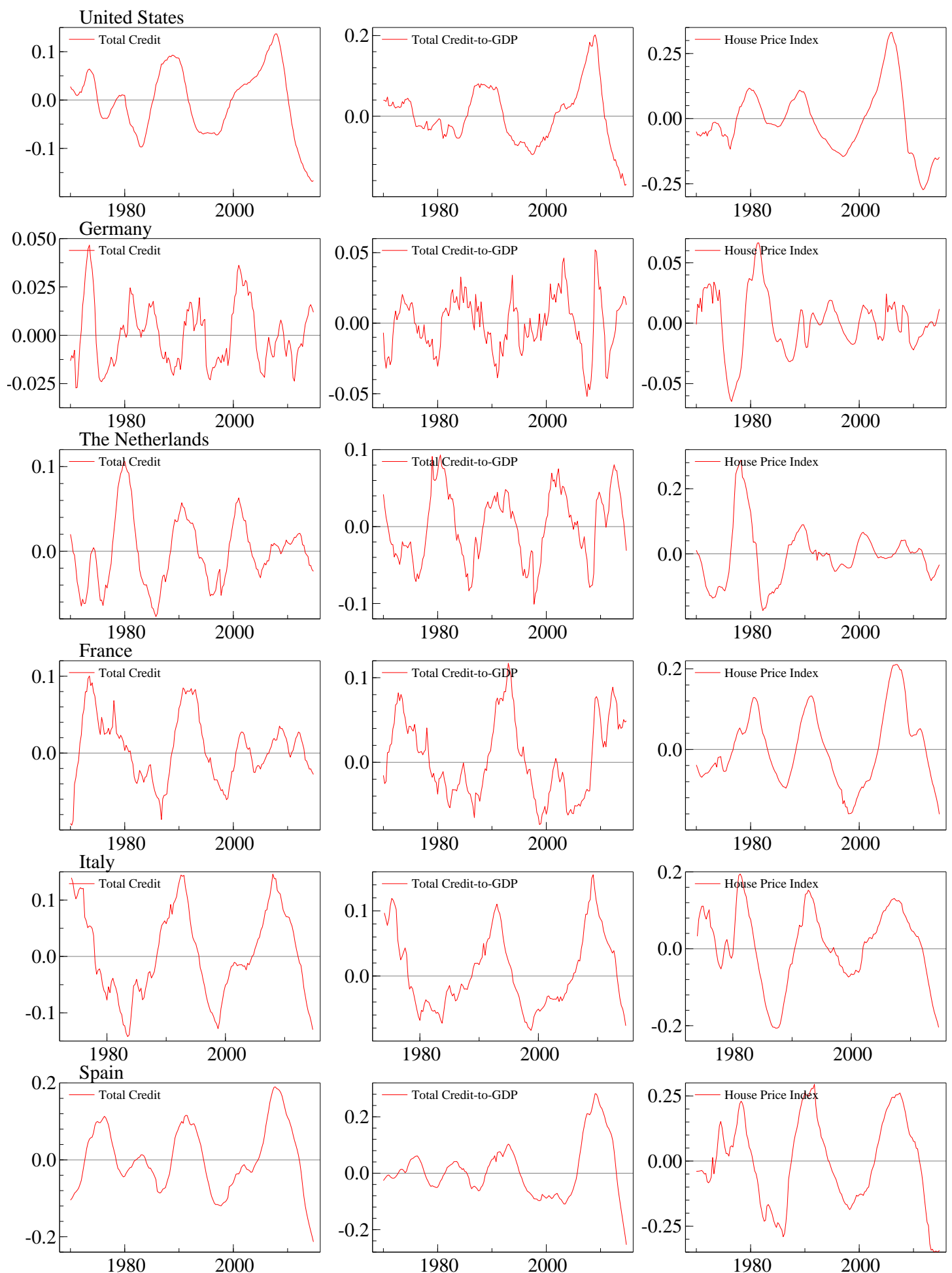
domain properties of the financial cycles are similar. In a joint multivariate UCTSM where similar cycles are imposed, we do not find statistical evidence that this more parsimonious model must be rejected. The reported statistics in Table 1 indicate that imposing similar cycles still provides a good fit of the data. Finally, without presenting all the evidence here (but it is available upon request) total credit and bank credit cycles co-move strongly within the EA, with comparable lengths and amplitudes. However, for the US, the two credit cycles have different dynamics. For example, bank credit-to-GDP ratio does not have strong trend fluctuations while total credit-to-GDP ratio exhibits an upward trend; see Figure 1. This result is consistent with financial markets playing a key role in financial intermediation in the US, see the discussions in Dembiermont et al. (2013) and Errico et al. (2014). In the EA, almost all credit is supplied by banks.

\section{Conclusions}

In this paper we explore a model-based methodology to extract trends and financial cycles for the US and the EA. Our analysis shows that credit, the credit-to-GDP ratio and real house prices exhibit similar medium-term cyclical behaviour with ample fluctuations. We find that the persistence, length and amplitude of the extracted cycles vary over time and vary across countries. In particular, we report differences in the financial cycles within the EA and we establish that US financial cycles have increased in amplitude and persistence from the mid-1980s.

\section{References}

Aikman, D., A. G. Haldane, and B. D. Nelson (2015). Curbing the Credit Cycle. The Economic Journal 125(585), 1072-1109.

Borio, C. (2014). The financial cycle and macroeconomics: What have we learnt? Journal of Banking \& Finance $45(\mathrm{C}), 182-198$.

Borio, C., C. Furfine, and P. Lowe (2001). Procyclicality of the financial system and financial stability: issues and policy options. In B. for International Settlements (Ed.), Marrying the macro- and micro-prudential dimensions of financial stability, Volume 1 of BIS Papers Chapters, pp. 1-57. Bank for International Settlements.

Burns, A. F. and W. C. Mitchell (1946). Measuring Business Cycles. Number burn46-1 in NBER Books. National Bureau of Economic Research, Inc.

Chen, X., A. Kontonikas, and A. Montagnoli (2012). Asset prices, credit and the business cycle. Economic Letters 117, 857-861.

Claessens, S., M. A. Kose, and M. E. Terrones (2011). Financial Cycles: What? How? When? NBER International Seminar on Macroeconomics 7(1), 303 - 344.

Claessens, S., M. A. Kose, and M. E. Terrones (2012). How do business and financial cycles interact? Journal of International Economics 87(1), 178-190. 
Dembiermont, C., M. Drehmann, and S. Muksakunratana (2013). How much does the private sector really borrow - a new database for total credit to the private non-financial sector. BIS Quarterly Review.

Drehmann, M., C. Borio, and K. Tsatsaronis (2012). Characterising the financial cycle: don't lose sight of the medium term! BIS Working Papers 380, Bank for International Settlements.

Durbin, J. and S. J. Koopman (2012). Time series analysis by state space methods (2nd ed.). Oxford University Press.

Errico, L., A. Harutyunyan, E. Loukoianova, R. Walton, Y. Korniyenko, G. Amidzic, H. AbuShanab, and H. S. Shin (2014). Mapping the Shadow Banking System Through a Global Flow of Funds Analysi. IMF Working Paper 14/10, International Monetary Fund.

Harvey, A. C. (1989). Forecasting, structural time series models and the Kalman filter. Cambridge University Press.

Harvey, A. C. and S. J. Koopman (1997). Multivariate Structural Time Series Models. In C. Heij, H. Schumacher, B. Hanzon, and C. Praagman (Eds.), System Dynamics in Economic and Financial Models. John Wiley and Sons Ltd.

Harvey, A. C. and T. M. Trimbur (2003). General Model-Based Filters for Extracting Cycles and Trends in Economic Time Series. The Review of Economics and Statistics 85(2), 244255 .

Hiebert, P. P., B. Klaus, T. A. Peltonen, Y. S. Schüler, and P. Welz (2014). Capturing the financial cycle in euro area countries. In Financial Stability Review November 2014. European Central Bank.

Igan, D., A. N. Kabundi, F. N.-D. Simone, M. Pinheiro, and N. T. Tamirisa (2009). Three Cycles; Housing, Credit, and Real Activity. IMF Working Papers 09/231, International Monetary Fund.

Koopman, S. J. and A. Lucas (2005). Business and default cycles for credit risk. Journal of Applied Econometrics 20(2), 311-323.

Schüler, Y. S., P. P. Hiebert, and T. A. Peltonen (2015). Characterising the financial cycle: a multivariate and time-varying approach. In ECB Working Paper 1846. European Central Bank.

Valle e Azevedo, J., S. J. Koopman, and A. Rua (2006). Tracking the Business Cycle of the Euro Area: A Multivariate Model-Based Bandpass Filter. Journal of Business and Economic Statistics 24, 278-290.

Zarnowitz, V. and A. Ozyildirim (2002). Time Series Decomposition and Measurement of Business Cycles, Trends and Growth Cycles. NBER Working Paper 8736, National Bureau of Economic Research, Inc. 